\title{
Effect of Applying Organic Amendments and Chickpea Integration on Soil Chemical Properties in Different Cropping Systems in Central Kenya
}

\author{
O. H. Ndukhu ${ }^{1}$, G. R. Wahome ${ }^{1} \&$ H. H. Jensen ${ }^{2}$ \\ ${ }^{1}$ University of Nairobi, Kenya \\ ${ }^{2}$ National Food Institute, Technical University of Denmark, Denmark \\ Correspondence: O. H. Ndukhu, University of Nairobi, Kenya. E-mail: hndukhu@yahoo.com \\ . N. Nakhu', G. R. Wahome' \& H. H. Jensen
}

Received: May 28, 2018

doi:10.5539/jas.v10n12p215

Accepted: September 10, $2018 \quad$ Online Published: November 15, 2018

URL: https://doi.org/10.5539/jas.v10n12p215

\begin{abstract}
A study was carried out to determine the effect of applying farm yard manure (FYM) and Minjingu rock phosphate (MRP) on soil available nitrogen, phosphorus and organic carbon. The study involved field experiments under varying precipitation pattern, soil fertility levels and cropping systems over four growth seasons. The experimental design was a randomized complete block (RCBD) with four replications in a split plot arrangement where the main plots were the three cropping systems; monocropping, intercropping and crop rotation and the split plots were FYM and MRP and sampling done at crop physiological maturity. Soil pH, N, P $\mathrm{K}$ and $\mathrm{C}$ increased in the different treatments in the following order control $<\mathrm{MRP}<\mathrm{FYM}$ in the three cropping systems across the four growing seasons at both sites. In maize under rotation with chickpea control had; $0.281 \%$ N, 2.82\% C and 10.68 ppm P. FYM; 0.554\% N, 4.41\% C and 18.24 ppm P. MRP; 0.45\% N, 3.6\% C and 41.08 ppm P. Maize chickpea intercrop control; 0.389\% N, 3.192\% C and 13.4 ppm P. FYM; $0.531 \%$ N, $4.98 \%$ C and 41.02 ppm P. MRP; $0.49 \%$ N, 4.08\% C and 50.9 ppm P. Soil under maize monocrop exhibited; control; $0.2 \%$ N, $2.59 \% \mathrm{C}$ and 11.26 ppm P. FYM; $0.416 \% \mathrm{~N}, 3.83 \% \mathrm{C}$ and $18.01 \mathrm{ppm}$ P. MRP; $0.28 \% \mathrm{~N}, 3.13 \% \mathrm{C}$ and $26.1 \mathrm{ppm}$ P. Almost a similar trend was observed in maize and tomato plots at both sites in all the growing seasons. Thus it can be deduced that, FYM and MRP application and legume integration in cropping systems improves soil fertility.
\end{abstract}

Keyswords: organic cropping systems, organic inputs, soil nutrient dynamics

\section{Introduction}

Several organic materials have been reported as suitable soil amendments for increasing crop production (Makinde \& Ayoola, 2010; Lehmann \& Joseph, 2009; Bonanomi et al., 2010). Naturally occurring organic fertilisers include manure slurry, worm castings, peat, seaweed, sewage and guano (Miles \& Manson, 2005). The potential of cow dung, poultry droppings, refuse compost and farmyard manure as suitable soil amendments with fertiliser value in the tropics has been well studied (Makinde \& Ayoola, 2010; Amanullah et al., 2010; Rufino et al., 2006; Das et al., 2012). Application of organic materials as fertilisers provides growth regulating substances and improves the physical, chemical and microbial properties of soil for plant growth (Lal, 2003). Plants need nitrogen, phosphorus, and potassium, as well as micronutrients and symbiotic relationships with fungi and other organisms to flourish, but getting enough nitrogen, and particularly synchronization so that plants get enough nitrogen when they need it most, is likely the greatest challenge for organic farmers (Makinde \& Ayoola, 2010; Tunstall, 2008). Organic farmers also use animal manure, certain processed fertilizers such as seed meal and various mineral powders such as indigenous rock phosphate (Jama \& Van Straaten, 2006; Van Straaten, 2002) and greensand, a naturally occurring form of potash which provides potassium, to maintain soil fertility (Lal, 2003). Among the common organic manures in use in fertility management of soils in Kenya are poultry manure, cattle dung, swine dung and goat dung. The use of cattle dung by farmers cuts across the country. Research on soil and soil organisms has proven beneficial to organic farming (Chen, 2006; Hole et al., 2005; Ramesh et al., 2005).

Varieties of bacteria and fungi break down plant matter and animal waste into productive soil nutrients (Lal, 2003). In turn, they produce benefits of safer yields and more productive soil for future crops (Ingram, 2007). 
Apart from supplying nutrients, manure maintains soil organic matter (Riley et al., 2008; Mando et al., 2005). However, the uses of organic manures as sources of soil improvement are hindered by its bulkiness, low nutrients quality and low mineralization rates (Ayeni, 2010). Integrated soil nutrition management has been more feasible in maintaining nutrients status as well as crop production than single application of mineral or organic fertilizers (Vanlauwe et al., 2005). Thus Ayeni (2010) advocated for the use of combined organic waste and rock phosphates in legume integrated cropping systems.

The objective of this study was to investigate the effect of application of farm yard manure and Minjingu rock phosphate on soil available nitrogen, phosphorus and organic carbon under field conditions under two crops (tomato and maize) intercropped with chickpea in soils typical of central Kenya.

\section{Materials and Methods}

\subsection{Site Description}

The study was conducted both on-farm (one farmer's field in Kiserian, Kajiado County) and on-station at Kabete Campus field station, University of Nairobi, Kenya. The Kiserian site lies at S $01.42254^{\circ}$ and E $036.66036^{\circ}$ with an elevation of $2070 \mathrm{~m}$ a.s.l and categorized under agro-ecological zone IV (Sombreak et al., 1982). The climate is typically semi-arid with annual temperature from $20^{\circ} \mathrm{C}$ and $28{ }^{\circ} \mathrm{C}$ with a mean of $25^{\circ} \mathrm{C}$ and a total annual rainfall between 450 and 1200mm (Braunn \& Weg, 1977). Kiserian soil is phaeozems (FAO-UNESCO, 1990). The Kabete site lies between S $01.24356^{\circ}$, E $036.74186^{\circ}$ with an elevation of $1856 \mathrm{~m}$ a.s.l. and categorized under agro-ecological zone III (Sombreak et al., 1982). The climate is typically sub-humid with mean annual temperature varying from $12-23{ }^{\circ} \mathrm{C}$ and a total annual rainfall ranging between 1200 and $1800 \mathrm{~mm}$ (Braunn \& Weg, 1977). Soil type is nitosols (FAO-UNESCO, 1990). Rainfall is bimodal with the long rains starting from March to June and short rains from October to December.

\subsection{Treatments and Experimental Design}

The experimental design was a randomized complete block design (RCBD) with split plot arrangement replicated four times. The main plots were cropping systems; monocropping, intercropping and crop rotation and the split plots were inputs (FYM and MPR). Each plot measured $4.8 \times 3.75 \mathrm{~m}$. The test crops were; tomato (Lycopersicon esculentum L., variety Rio Grande) and maize (Zea mays L, var. H513), intercropped or in rotation with chickpea.

\subsection{Land Preparation, Planting and Weeding of Tomato, Maize and Chickpea}

The land was prepared manually using hand hoes late February and September of 2012 and 2013. A nursery was established for tomato seed germination and after 6 weeks seedlings were transplanted each season. FYM characterization was done to determine the application rate to supply enough $\mathrm{C}, \mathrm{N}$ and P. Often $10 \mathrm{t} \mathrm{ha}^{-1} \mathrm{FYM}$ has been used in several field experiments to supply adequate amounts of $\mathrm{P}$ and N. Since Minjingu phosphate rock contains $28 \% \mathrm{P}_{2} \mathrm{O}_{5}, 490 \mathrm{~kg} \mathrm{ha}^{-1}$ of it was applied to supply the recommended $60 \mathrm{~kg} \mathrm{P}^{-1}$ to obtain good crop yields. Spacing of $30 \times 75 \mathrm{~cm}$ for maize, $45 \times 75 \mathrm{~cm}$ for tomatoes and $10 \times 30 \mathrm{~cm}$ for chickpea pure stands were adopted. Weeding was done at 3 weeks after transplanting and after fruiting. Biopesticides and local plant extracts were used in pests and diseases management. The crops were planted in March and October during the long and short rain seasons of the years 2012 and 2013 and laid out as shown below (Figure 1).

\begin{tabular}{|c|c|c|c|c|c|c|c|c|}
\hline \multicolumn{3}{|c|}{ Monocropping } & \multicolumn{3}{|c|}{ Crop rotation } & \multicolumn{3}{|c|}{ Intercropping } \\
\hline FYM & MPR & Control & MPR & FYM & Control & FYM & MPR & Control \\
\hline \multicolumn{3}{|c|}{ Intercropping } & \multicolumn{3}{|c|}{ Monocropping } & \multicolumn{3}{|c|}{ Crop Rotation } \\
\hline MPR & Control & FYM & FYM & Control & MPR & Control & FYM & MPR \\
\hline \multicolumn{3}{|c|}{ Crop rotation } & \multicolumn{3}{|c|}{ Monocropping } & \multicolumn{3}{|c|}{ Intercropping } \\
\hline Control & MPR & FYM & Control & FYM & MPR & FYM & Control & MPR \\
\hline \multicolumn{3}{|c|}{ Intercropping } & \multicolumn{3}{|c|}{ Crop rotation } & \multicolumn{3}{|c|}{ Monocropping } \\
\hline FYM & MPR & Control & MPR & FYM & Control & FYM & MPR & Control \\
\hline
\end{tabular}

Figure 1. Field layout 
Table 1. Effects of cropping systems and organic inputs on soil nutrient status in maize at Kabete

\begin{tabular}{|c|c|c|c|c|c|c|c|}
\hline Season & Cropping system & Organic inputs & $\mathrm{pH}\left(\mathrm{H}_{2} \mathrm{O}\right)$ & N (\%) & $\mathrm{C}(\%)$ & $\mathrm{P}(\mathrm{ppm})$ & $\mathrm{K}\left(\mathrm{Cmol} \mathrm{kg}^{-1}\right)$ \\
\hline \multirow{9}{*}{ I } & \multirow{3}{*}{ Crop rotation } & CONT & $4.69^{\text {cde }}$ & $0.26^{\mathrm{cd}}$ & $2.81^{\mathrm{c}}$ & $12.2^{\mathrm{bc}}$ & $0.72^{\text {hij }}$ \\
\hline & & FYM & $5.39^{\mathrm{klmn}}$ & $0.50^{\mathrm{op}}$ & $4.15^{\mathrm{k}}$ & $19.2^{\mathrm{f}}$ & $1.04^{\mathrm{s}}$ \\
\hline & & MRP & $4.91^{\mathrm{efg}}$ & $0.32^{\text {fgh }}$ & $3.39^{\mathrm{g}}$ & $28.2^{\mathrm{h}}$ & $0.85^{\mathrm{m}}$ \\
\hline & \multirow{3}{*}{ Intercrop } & CONT & $4.73^{\text {cdef }}-$ & $0.35^{\mathrm{hij}-}$ & $2.85^{\mathrm{c}}$ & $11.4^{\mathrm{abc}-}$ & $0.62^{\mathrm{e}^{-}}$ \\
\hline & & FYM & $5.42^{\mathrm{klmn}}$ & $0.48^{\text {no }}$ & $4.45^{1}$ & $35.0^{\mathrm{i}}$ & $0.92^{\mathrm{pq}}$ \\
\hline & & MRP & $5.34^{\mathrm{klmn}}$ & $0.44^{\mathrm{m}}$ & $3.63^{\mathrm{h}}$ & $43.4^{\mathrm{k}}$ & $0.79^{1}$ \\
\hline & \multirow{3}{*}{ Monocrop } & CONT & $4.31^{\mathrm{a}-\cdots}$ & $0.16^{\mathrm{a}}$ & $2.63^{\mathrm{a} \bar{b}^{-}}$ & $11.0^{\mathrm{abc} c^{-1}}$ & $0.40^{\mathrm{a}}$ \\
\hline & & FYM & $4.95^{\mathrm{efgh}}$ & $0.34^{\text {ghi }}$ & $4.10^{\mathrm{k}}$ & $17.6^{\mathrm{ef}}$ & $0.67^{\mathrm{f}}$ \\
\hline & & MRP & $4.52^{\mathrm{abc}}$ & $0.23^{\mathrm{bc}}$ & $3.35^{\mathrm{g}}$ & $25.5^{\mathrm{g}}$ & $0.46^{\mathrm{bc}}$ \\
\hline \multirow{9}{*}{ II } & \multirow{3}{*}{ Crop rotation } & CONT & $4.59^{\mathrm{bcd}}$ & $0.28^{\text {def }}$ & $2.74^{\mathrm{bc}}$ & $9.7^{\mathrm{ab}}$ & $0.70^{\text {fgh }}$ \\
\hline & & FYM & $5.25^{\mathrm{jklm}}$ & $0.56^{\mathrm{q}}$ & $4.40^{1}$ & $16.5^{\mathrm{e}}$ & $1.05^{\mathrm{s}}$ \\
\hline & & MRP & $5.18^{\text {hijk }}$ & $0.36^{\text {hijk }}$ & $3.28^{\mathrm{fg}}$ & $37.2^{\mathrm{ij}}$ & $0.89^{\text {nop }}$ \\
\hline & \multirow{3}{*}{ Intercrop } & CONT & $4.78^{\text {cde } \overline{f g}}-$ & $0.35^{\text {ghij }}$ & $3.15^{\mathrm{de}}$ & $12.1^{\mathrm{bc}}$ & $0.68^{\mathrm{fg}-1}$ \\
\hline & & FYM & $5.47^{1 \mathrm{mn}}$ & $0.53^{\mathrm{pq}}$ & $4.65^{\mathrm{m}}$ & $37.1^{\mathrm{ij}}$ & $0.94^{\mathrm{q}}$ \\
\hline & & MRP & $5.39^{\mathrm{klmn}}$ & $0.45^{\mathrm{m}}$ & $3.80^{\mathrm{ij}}$ & $46.1^{1}$ & $0.78^{\mathrm{kl}}$ \\
\hline & \multirow{3}{*}{ Monocrop } & CONT & $4.55^{\mathrm{a} \bar{c} \mathrm{~d}}{ }^{-}$ & $0.18^{\mathrm{a}}$ & $2.56^{\mathrm{a}}$ & $9.1^{\mathrm{a}-}$ & $0.43^{\mathrm{a}-}$ \\
\hline & & FYM & $5.22^{\mathrm{ijkl}}$ & $0.37^{\mathrm{ijk}}$ & $4.10^{\mathrm{k}}$ & $15.6^{\mathrm{de}}$ & $0.71^{\text {ghi }}$ \\
\hline & & MRP & $4.76^{\mathrm{cdefg}}$ & $0.26^{\mathrm{cd}}$ & $3.06^{\mathrm{d}}$ & $35.0^{\mathrm{i}}$ & $0.49^{\mathrm{cd}}$ \\
\hline \multirow{9}{*}{ III } & \multirow{3}{*}{ Crop rotation } & CONT & $4.73^{\text {cdef }}$ & $0.32^{\text {efgh }}$ & $2.77^{\mathrm{c}}$ & $9.9^{\mathrm{ab}}$ & $0.74^{\mathrm{ij}}$ \\
\hline & & FYM & $5.44^{\mathrm{klmn}}$ & $0.56^{\mathrm{pq}}$ & $4.45^{1}$ & $16.9^{\mathrm{ef}}$ & $1.10^{\mathrm{t}}$ \\
\hline & & MRP & $4.96^{\mathrm{efgh}}$ & $0.42^{\mathrm{lm}}$ & $3.31^{\mathrm{fg}}$ & $38.0^{\mathrm{j}}$ & $0.86^{\mathrm{mn}}$ \\
\hline & \multirow{3}{*}{ Intercrop } & CONT & $4.75^{\text {cdefg }^{--}}$ & $0.31^{\mathrm{efg}-}$ & $3.10^{\mathrm{de}^{-}}$ & $12.4^{\mathrm{bc}^{--}}$ & $0.69^{\mathrm{fgh}}$ \\
\hline & & FYM & $5.46^{\mathrm{lmn}}$ & $0.62^{\mathrm{r}}$ & $4.98^{\mathrm{n}}$ & $38.0^{\mathrm{j}}$ & $0.99^{r}$ \\
\hline & & MRP & $5.77^{\circ}$ & $0.39^{\mathrm{kl}}$ & $3.71^{\mathrm{hi}}$ & $47.1^{1}$ & $0.80^{1}$ \\
\hline & \multirow{3}{*}{ Monocrop } & CONT & $4.37^{\mathrm{ab}^{--}}$ & $0.18^{\mathrm{a}}$ & $2.78^{\mathrm{c}-}$ & $10.4^{\mathrm{a}^{-}}$ & $0.44^{\mathrm{b}}$ \\
\hline & & FYM & $5.02^{\text {ghij }}$ & $0.38^{\mathrm{ijk}}$ & $4.11^{\mathrm{k}}$ & $16.6^{\mathrm{e}}$ & $0.73^{\mathrm{ij}}$ \\
\hline & & MRP & $5.31^{\mathrm{klm}}$ & $0.26^{\mathrm{cd}}$ & $3.36^{\mathrm{g}}$ & $24.0^{\mathrm{g}}$ & $0.50^{\mathrm{d}}$ \\
\hline \multirow{11}{*}{ IV } & \multirow{3}{*}{ Crop rotation } & CONT & $4.61^{\mathrm{bcd}}$ & $0.28^{\mathrm{de}}$ & $2.82^{\mathrm{c}}$ & $10.7^{\mathrm{abc}}$ & $0.71^{\text {ghi }}$ \\
\hline & & FYM & $5.29^{\mathrm{klm}}$ & $0.55^{\mathrm{q}}$ & $4.40^{1}$ & $18.2^{\text {ef }}$ & $1.10^{\mathrm{t}}$ \\
\hline & & MRP & $5.60^{\mathrm{no}}$ & $0.45^{\mathrm{mn}}$ & $3.60^{\mathrm{h}}$ & $41.1^{\mathrm{k}}$ & $0.90^{\mathrm{op}}$ \\
\hline & \multirow{3}{*}{ Intercrop } & CONT & $4.8^{\operatorname{defg}^{--}}$ & $0.39^{\mathrm{jk \textrm {I } ^ { - }}}$ & $3.19^{\mathrm{ef}^{-}}$ & $13.4^{\mathrm{cd}^{-1}}$ & $0.69^{\mathrm{fgh}^{--}}$ \\
\hline & & FYM & $5.51^{\mathrm{mn}}$ & $0.53^{\mathrm{pq}}$ & $4.98^{\mathrm{n}}$ & $41.0^{\mathrm{k}}$ & $1.04^{\mathrm{s}}$ \\
\hline & & MRP & $5.83^{\circ}$ & $0.46^{\text {no }}$ & $4.07^{\mathrm{k}}$ & $50.9^{\mathrm{m}}$ & $0.88^{\mathrm{mno}}$ \\
\hline & \multirow{3}{*}{ Monocrop } & CONT & $4.35^{\mathrm{ab}^{-1}}$ & $0.20^{\mathrm{a}{ }^{-}}$ & $2.59^{\mathrm{a}}$ & $11.3^{\mathrm{ab} \bar{c}^{-1}}$ & $0.45^{\mathrm{b}^{--}}$ \\
\hline & & FYM & $4.98^{\text {fghi }}$ & $0.42^{\mathrm{lm}}$ & $3.83^{\mathrm{j}}$ & $18.0^{\mathrm{ef}}$ & $0.75^{\mathrm{jk}}$ \\
\hline & & MRP & $4.91^{\mathrm{efg}}$ & $0.29^{\mathrm{def}}$ & $3.13^{\mathrm{de}}$ & $26.1^{\mathrm{gh}}$ & $0.51^{\mathrm{d}}$ \\
\hline & & Mean & 5.03 & 0.37 & 3.56 & 24.06 & 0.75 \\
\hline & & $\mathrm{LSD}_{0.05}$ & 0.23 & 0.03 & 0.11 & 2.36 & 0.03 \\
\hline
\end{tabular}

Note. Cont-control, MRP-Minjingu rock phosphate, FYM-farm yard manure. Means with same letters within the column are not significantly different $(\mathrm{P}<0.05)$.

\subsection{Soil Sampling and Analysis}

Soil sampling was done at planting and harvesting of the crops of each season. Soil samples were collected using a soil auger at a depth of $0-20 \mathrm{~cm}$ at 5 points using W-sampling procedure as described by Peters et al. (2008). The soil in each experimental unit was composited and a $500 \mathrm{~g}$ sample obtained and bagged in polythene bags for $\mathrm{pH}, \mathrm{P}, \mathrm{K}$, total N, and organic-C analyses at the Section of Soil Science, Department of Land Resource Management and Agricultural Technology (LARMAT) at the University of Nairobi.

The $\mathrm{pH}$ was measured with a glass electrode $\mathrm{pH}$ meter on 1: $2.5(\mathrm{w} / \mathrm{v})$ suspension of soil in water, and on $0.01 \mathrm{M}$ $\mathrm{CaCl}_{2}$ solution, in all cases after shaking for 30 minutes (Okalebo et al., 2002). The plant available soil $\mathrm{P}$ content was estimated after shaking for 30 minutes at 1:10 ratio with double acid (conc. $\mathrm{HCl}$ and $\mathrm{H}_{2} \mathrm{SO}_{4}$ ). The Molybdenum Blue method was followed (Mehlich et al., 1962). The organic carbon was estimated by the 
Walkley-Black method (Black, 1965). The total nitrogen was measured by the semi-micro Kjeldahl method (Black, 1965), and $\mathrm{K}\left(\mathrm{Cmol} \mathrm{kg}^{-1}\right)$ was determined using flame photometer (Dean, 1960).

\subsection{Statistical Analysis}

Analysis of variance to assess the effects of sites, seasons, cropping systems and organic inputs (treatments) and their interactions on soil chemical properties was conducted using GENSTAT $15^{\text {th }}$ Edition (Payne et al., 2006). The Least Significant Difference (LSD) test was used to separate means of significant differences among treatment means $(\mathrm{P}<0.05)$ (Steel \& Torrie, 1987).

\section{Results and Discussion}

\subsection{Effects of Cropping Systems and Organic Inputs on Soil Nutrient Status in Maize}

The addition of FYM and MRP improved significantly $(\mathrm{p}<0.05)$ soil fertility in teams of $\mathrm{pH}, \mathrm{N}, \mathrm{P}, \mathrm{K}$ and $\mathrm{C}$ compared to a control treatment with no inputs across seasons and cropping systems. Soil pH, N, P K and C increased in the different treatments in the following order control $<$ MRP $<$ FYM in the three cropping systems across the four growing seasons at both sites. That is, MRP and FYM application had a significant $(\mathrm{p}<0.05)$ effect on soil nutrient contents. The results of soil analysis in the maize at Kabete are shown in Table 1 and those of the Kiserian site in Table 2. Continuous cropping of land with little or no nutrient returns in the control plots have been found to lead to nutrient depletion and decline in soil fertility (Smalling et al., 1997; Bationo et al., 2007; Giller et al., 2009; Miao et al., 2010).

The increase in yield found in the current study is therefore attributed to the increased available nutrients due to MRP and FYM application. The higher nutrient uptake with combined FYM, MPR and legume integrations than the control in monocropping was probably synergised by enhanced $P$ uptake (Teng \& Timmer, 1994). Increased fertilizer $\mathrm{N}$ and $\mathrm{P}$ application depleting soil levels of other nutrients (such as $\mathrm{K}, \mathrm{Ca}, \mathrm{Mg}$ and $\mathrm{Zn}$ ) were obtained by Smalling et al. (1997) and explained as due to rapid crop removal. Manure application increases soil pH, N, P, and such cations as $\mathrm{Ca}$ and $\mathrm{Mg}$ due to direct crop responses or to physical effects of addition of soil organic matter on water filtration or retention (Cooperband, 2002). However, the responses to cattle manure application are highly variable just as the chemical composition of the manures. Storage conditions may result in ammonia loss through volatilization and leaching of nitrates. Differences in cattle diets in addition to method of collection and storage, degree of decomposition and handling conditions also influence manure levels of organic $\mathrm{C}$ and $\mathrm{N}$. A survey in Kisii central district in Kenya to determine how livestock and manure management practices (stocking rate, feeding, collection, composition and storage) affected the manure for crop production, indicated that collecting and heaping kraal manure on the soil surface resulted in very low quality manure (Schnier et al., 1996). 
Table 2. Effects of cropping systems and organic inputs on soil nutrient status in maize at Kiserian

\begin{tabular}{|c|c|c|c|c|c|c|c|}
\hline Season & Cropping system & Organic inputs & $\mathrm{pH}\left(\mathrm{H}_{2} \mathrm{O}\right)$ & N (\%) & $\mathrm{C}(\%)$ & $\mathrm{P}(\mathrm{ppm})$ & $\mathrm{K}\left(\mathrm{Cmol} \mathrm{kg}^{-1}\right)$ \\
\hline \multirow{9}{*}{ I } & \multirow{3}{*}{ Crop rotation } & CONT & $4.45^{\text {cde }}$ & $0.24^{\text {cd }}$ & $2.67^{\mathrm{c}}$ & $11.6^{\mathrm{bc}}$ & $0.69^{\text {hij }}$ \\
\hline & & FYM & $5.12^{\mathrm{klmn}}$ & $0.48^{\mathrm{op}}$ & $3.94^{\mathrm{k}}$ & $18.5^{\mathrm{f}}$ & $0.98^{\mathrm{s}}$ \\
\hline & & MRP & $4.66^{\mathrm{efg}}$ & $0.31^{\mathrm{fgh}}$ & $3.22^{\mathrm{g}}$ & $26.8^{\mathrm{h}}$ & $0.81^{\mathrm{m}}$ \\
\hline & \multirow{3}{*}{ Intercrop } & CONT & $4.50^{\text {cdef }^{-1}}$ & $0.34^{\text {hij }}$ & $2.71^{c^{-}}$ & $10.9^{\mathrm{abc}-1}$ & $0.58^{\mathrm{e}^{-}}$ \\
\hline & & FYM & $5.14^{\mathrm{klmn}}$ & $0.46^{\text {no }}$ & $4.22^{1}$ & $33.2^{\mathrm{i}}$ & $0.88^{\mathrm{pq}}$ \\
\hline & & MRP & $5.07^{\mathrm{klmn}}$ & $0.42^{\mathrm{m}}$ & $3.45^{\mathrm{h}}$ & $41.2^{\mathrm{k}}$ & $0.75^{1}$ \\
\hline & \multirow{3}{*}{ Monocrop } & CONT & $4.10^{\mathrm{a}}$ & $0.16^{\mathrm{a}}$ & $2.50^{\mathrm{ab}}$ & $10.5^{\mathrm{a} \bar{b} c^{-}}$ & $0.38^{\mathrm{a}}$ \\
\hline & & FYM & $4.71^{\mathrm{efgh}}$ & $0.32^{\text {ghi }}$ & $3.90^{\mathrm{k}}$ & $16.7 \mathrm{ef}$ & $0.63^{\mathrm{f}}$ \\
\hline & & MRP & $4.29^{\mathrm{abc}}$ & $0.22^{\mathrm{bc}}$ & $3.18^{\mathrm{g}}$ & $24.2^{\mathrm{g}}$ & $0.44^{\mathrm{bc}}$ \\
\hline \multirow{9}{*}{ II } & \multirow{3}{*}{ Crop rotation } & CONT & $4.36^{\mathrm{bcd}}$ & $0.27^{\mathrm{def}}$ & $2.60^{\mathrm{bc}}$ & $9.2^{\mathrm{ab}}$ & $0.66^{\mathrm{fgh}}$ \\
\hline & & FYM & $4.99^{\mathrm{jklm}}$ & $0.53^{\mathrm{q}}$ & $4.18^{1}$ & $15.7^{\mathrm{e}}$ & $0.99^{\mathrm{s}}$ \\
\hline & & MRP & $4.92^{\mathrm{hijk}}$ & $0.34^{\mathrm{hijk}}$ & $3.11^{\mathrm{fg}}$ & $35.3^{\mathrm{ij}}$ & $0.85^{\text {nop }}$ \\
\hline & \multirow{3}{*}{ Intercrop } & CONT & $4.54^{\text {cdefg }}$ & $0.33^{\text {ghij }}$ & $2.99^{\mathrm{de}}$ & $11.5^{\mathrm{bc}}$ & $0.65^{\mathrm{fg}^{--}}$ \\
\hline & & FYM & $5.20^{\mathrm{lmn}}$ & $0.50^{\mathrm{pq}}$ & $4.41^{\mathrm{m}}$ & $35.3^{\mathrm{ij}}$ & $0.90^{\mathrm{q}}$ \\
\hline & & MRP & $5.12^{\mathrm{klmn}}$ & $0.42^{\mathrm{m}}$ & $3.61^{\mathrm{ij}}$ & $43.8^{1}$ & $0.74^{\mathrm{kl}}$ \\
\hline & \multirow{3}{*}{ Monocrop } & CONT & $4.32^{\mathrm{abcd}}$ & $0.17^{\mathrm{a}-}$ & $2.43^{\mathrm{a}}$ & $8.7^{\mathrm{a}-1}$ & $0.41^{\mathrm{ab}^{--}}$ \\
\hline & & FYM & $4.96^{\mathrm{ijkl}}$ & $0.36^{\mathrm{ijk}}$ & $3.90^{\mathrm{k}}$ & $14.8^{\mathrm{de}}$ & $0.68^{\text {ghi }}$ \\
\hline & & MRP & $4.52^{\text {cdefg }}$ & $0.24^{\mathrm{cd}}$ & $2.90^{\mathrm{d}}$ & $33.3^{\mathrm{i}}$ & $0.46^{\mathrm{cd}}$ \\
\hline \multirow{9}{*}{ III } & \multirow{3}{*}{ Crop rotation } & CONT & $4.50^{\text {cdef }}$ & $0.30^{\text {efgh }}$ & $2.63^{\mathrm{c}}$ & $9.4^{\mathrm{ab}}$ & $0.70^{\mathrm{ij}}$ \\
\hline & & FYM & $5.17^{\mathrm{klmn}}$ & $0.51^{\mathrm{pq}}$ & $4.22^{1}$ & $16.0^{\mathrm{ef}}$ & $1.04^{\mathrm{t}}$ \\
\hline & & MRP & $4.71^{\text {efgh }}$ & $0.40^{\mathrm{lm}}$ & $3.15^{\mathrm{fg}}$ & $36.1^{\mathrm{j}}$ & $0.82^{\mathrm{mn}}$ \\
\hline & \multirow{3}{*}{ Intercrop } & CONT & $4.52^{\text {cdéfg }}$ & $0.30^{\mathrm{efg}^{-}}$ & $2.95^{\mathrm{de}}$ & $11.8^{\mathrm{bc}^{-}}$ & $0.66^{\mathrm{fgh}}$ \\
\hline & & FYM & $5.18^{\mathrm{lmn}}$ & $0.59^{r}$ & $4.73^{\mathrm{n}}$ & $36.1^{\mathrm{j}}$ & $0.94^{\mathrm{r}}$ \\
\hline & & MRP & $5.48^{\circ}$ & $0.37^{\mathrm{kl}}$ & $3.52^{\text {hi }}$ & $44.8^{1}$ & $0.76^{1}$ \\
\hline & \multirow{3}{*}{ Monocrop } & CONT & $4.15^{\mathrm{ab}^{--}}$ & $0.17^{\mathrm{a}^{-}}$ & $2.64^{\mathrm{c}^{-}}$ & $9.9^{\mathrm{a} \mathrm{b}^{-1}}$ & $0.42^{b^{-}}$ \\
\hline & & FYM & $4.77^{\text {ghij }}$ & $0.36^{\mathrm{ijk}}$ & $3.90^{\mathrm{k}}$ & $15.8^{\mathrm{e}}$ & $0.70^{\mathrm{ij}}$ \\
\hline & & MRP & $5.04^{\mathrm{klm}}$ & $0.25^{\mathrm{cd}}$ & $3.19^{\mathrm{g}}$ & $22.8^{\mathrm{g}}$ & $0.48^{\mathrm{d}}$ \\
\hline \multirow{11}{*}{ IV } & \multirow{4}{*}{ Crop rotation } & CONT & $4.38^{\mathrm{bcd}}$ & $0.27^{\mathrm{de}}$ & $2.68^{\mathrm{c}}$ & $10.1^{\mathrm{abc}}$ & $0.67^{\text {ghi }}$ \\
\hline & & FYM & $5.03^{\mathrm{klm}}$ & $0.53^{\mathrm{q}}$ & $4.18^{1}$ & $17.3^{\text {ef }}$ & $1.05^{\mathrm{t}}$ \\
\hline & & MRP & $5.32^{\text {no }}$ & $0.43^{\mathrm{mn}}$ & $3.42^{\mathrm{h}}$ & $39.0^{\mathrm{k}}$ & $0.86^{\mathrm{op}}$ \\
\hline & & CONT & $4.56^{\text {defg }^{-}}$ & $0.37^{\mathrm{jk} \Gamma^{-}}$ & $3.03^{\mathrm{ef}}$ & $12.7^{\mathrm{cd}^{--}}$ & $0.65^{\mathrm{fgh}^{-}}$ \\
\hline & \multirow[t]{2}{*}{ Intercrop } & FYM & $5.23^{\mathrm{mn}}$ & $0.51^{\mathrm{pq}}$ & $4.73^{\mathrm{n}}$ & $39.0^{\mathrm{k}}$ & $0.99^{\mathrm{s}}$ \\
\hline & & MRP & $5.54^{\circ}$ & $0.46^{\text {no }}$ & $3.86^{\mathrm{k}}$ & $48.4^{\mathrm{m}}$ & $0.84^{\mathrm{mno}}$ \\
\hline & \multirow{3}{*}{ Monocrop } & CONT & $4.14^{\mathrm{ab}^{--}}$ & $0.19^{\mathrm{ab}^{-}}$ & $2.46^{\mathrm{a}}$ & $10.7^{\mathrm{a} \overline{\mathrm{b}} \mathrm{c}^{-}}$ & $0.43^{\mathrm{b}^{-}}$ \\
\hline & & FYM & $4.73^{\text {fghi }}$ & $0.40^{\mathrm{lm}}$ & $3.63^{\mathrm{j}}$ & $17.1^{\mathrm{ef}}$ & $0.71^{\mathrm{jk}}$ \\
\hline & & MRP & $4.66^{\mathrm{efg}}$ & $0.27^{\mathrm{def}}$ & $2.98^{\mathrm{de}}$ & $24.8^{\mathrm{gh}}$ & $0.49^{d}$ \\
\hline & & Mean & 4.78 & 0.35 & 3.38 & 22.86 & 0.712 \\
\hline & & $\mathrm{LSD}_{0.05}$ & 0.217 & 0.03 & 0.11 & 2.24 & 0.031 \\
\hline
\end{tabular}

Note. Cont-control, MRP-Minjingu rock phosphate, FYM-farm yard manure. Means with the same letters within the column are not significantly different $(\mathrm{P}<0.05)$. 
Table 3. Effects of cropping systems and organic inputs on soil nutrient status in tomato at Kabete

\begin{tabular}{|c|c|c|c|c|c|c|c|}
\hline Season & Cropping system & Organic inputs & $\mathrm{pH}\left(\mathrm{H}_{2} \mathrm{O}\right)$ & $\mathrm{N}(\%)$ & $\mathrm{C}(\%)$ & $\mathrm{P}(\mathrm{ppm})$ & $\mathrm{K}\left(\mathrm{Cmol} \mathrm{kg}^{-1}\right)$ \\
\hline \multirow{9}{*}{ I } & \multirow{3}{*}{ Crop rotation } & CONT & $4.11^{\text {bcd }}$ & $0.33^{\mathrm{d}}$ & $2.96^{\mathrm{c}}$ & $11.6^{\mathrm{bc}}$ & $0.64^{\text {hi }}$ \\
\hline & & FYM & $5.17^{\mathrm{lm}}$ & $0.45^{1}$ & $4.37^{\mathrm{k}}$ & $18.5^{\mathrm{f}}$ & $0.99^{\mathrm{q}}$ \\
\hline & & MRP & $4.68^{\text {ghi }}$ & $0.38^{\mathrm{fgh}}$ & $3.57^{\mathrm{g}}$ & $26.8^{\mathrm{h}}$ & $0.77^{\mathrm{m}}$ \\
\hline & \multirow{3}{*}{ Intercrop } & CONT & $4.24^{\mathrm{de}-}$ & $0.25^{\mathrm{b}}$ & $3.00^{\mathrm{c}}$ & $10.9^{\mathrm{a} b \mathrm{c}}$ & $0.48^{\mathrm{bc}}$ \\
\hline & & FYM & $5.75^{\mathrm{q}}$ & $0.63^{p}$ & $4.68^{1}$ & $33.3^{\mathrm{i}}$ & $1.12^{\mathrm{s}}$ \\
\hline & & MRP & $4.86^{\mathrm{ijk}}$ & $0.38^{\text {fgh }}$ & $3.82^{\mathrm{h}}$ & $41.3^{\mathrm{k}}$ & $0.56^{\mathrm{ef}}$ \\
\hline & \multirow{3}{*}{ Monocrop } & CONT & $3.97^{\mathrm{a} \mathrm{b}^{-1}}$ & $0.22^{\mathrm{a}}$ & $2.73^{\mathrm{a}}$ & $9.9^{\mathrm{a} \bar{b}^{--}}$ & $0.43^{\mathrm{a}}$ \\
\hline & & FYM & $5.01^{\mathrm{kl}}$ & $0.52^{\mathrm{n}}$ & $4.03^{\mathrm{j}}$ & $15.8^{\mathrm{e}}$ & $0.99^{\mathrm{q}}$ \\
\hline & & MRP & $4.55^{\mathrm{fg}}$ & $0.33^{\mathrm{d}}$ & $3.30^{\mathrm{de}}$ & $22.9^{\mathrm{g}}$ & $0.50^{\mathrm{cd}}$ \\
\hline \multirow{9}{*}{ II } & \multirow{3}{*}{ Crop rotation } & CONT & $4.18^{\text {bcde }}$ & $0.23^{\mathrm{a}}$ & $2.89^{b c}$ & $9.2^{\mathrm{ab}}$ & $0.68^{\mathrm{ij}}$ \\
\hline & & FYM & $5.27^{\mathrm{mn}}$ & $0.58^{\circ}$ & $4.63^{1}$ & $15.7^{\mathrm{e}}$ & $1.05^{\mathrm{r}}$ \\
\hline & & MRP & $4.78^{\mathrm{hij}}$ & $0.36^{\mathrm{efg}}$ & $3.45^{\mathrm{fg}}$ & $35.4^{\mathrm{ij}}$ & $0.82^{\mathrm{n}}$ \\
\hline & \multirow{3}{*}{ Intercrop } & CONT & $4.38^{\mathrm{e} \bar{f}^{-}}$ & $0.36^{\mathrm{efg}}$ & $3.31^{\mathrm{de}}$ & $11.6^{\mathrm{bc}}$ & $0.57^{-\mathrm{fg}}$ \\
\hline & & FYM & $5.52^{\mathrm{op}}$ & $0.50^{\mathrm{m}}$ & $4.89^{\mathrm{m}}$ & $35.4^{\mathrm{ij}}$ & $0.88^{\circ}$ \\
\hline & & MRP & $4.99^{\mathrm{jkl}}$ & $0.42^{\mathrm{jk}}$ & $4.00^{\mathrm{ij}}$ & $43.9^{1}$ & $0.73^{\mathrm{kl}}$ \\
\hline & \multirow{3}{*}{ Monocrop } & CONT & $3.86^{\mathrm{a}}$ & $0.28^{\mathrm{c}}$ & $2.69^{\mathrm{a}}$ & $10.5^{\mathrm{abc}}$ & $0.54^{\mathrm{def}^{-}}$ \\
\hline & & FYM & $4.86^{\mathrm{ijk}}$ & $0.41^{\mathrm{jk}}$ & $4.32^{\mathrm{k}}$ & $16.8^{\mathrm{ef}}$ & $0.83^{\mathrm{n}}$ \\
\hline & & MRP & $4.39^{\mathrm{ef}}$ & $0.35^{\mathrm{e}}$ & $3.22^{\mathrm{d}}$ & $24.3^{\mathrm{g}}$ & $0.69^{\mathrm{jk}}$ \\
\hline \multirow{9}{*}{ III } & \multirow{3}{*}{ Crop rotation } & CONT & $4.23^{\mathrm{de}}$ & $0.23^{\mathrm{a}}$ & $2.91^{\mathrm{c}}$ & $9.4^{\mathrm{ab}}$ & $0.69^{\mathrm{jk}}$ \\
\hline & & FYM & $5.33^{\mathrm{mno}}$ & $0.58^{\circ}$ & $4.68^{1}$ & $16.1^{\mathrm{ef}}$ & $1.07^{\mathrm{r}}$ \\
\hline & & MRP & $4.84^{\mathrm{ijk}}$ & $0.36^{\mathrm{ef}}$ & $3.49^{\mathrm{fg}}$ & $36.2^{\mathrm{j}}$ & $0.83^{\mathrm{n}}$ \\
\hline & \multirow{3}{*}{ Intercrop } & CONT & $4.51^{\mathrm{fg}}$ & $0.36^{\mathrm{efg}}$ & $3.26^{\mathrm{de}}$ & $11.8^{\mathrm{bc}-}$ & $0.58^{\mathrm{fg}}$ \\
\hline & & FYM & $5.68^{\mathrm{pq}}$ & $0.49^{\mathrm{m}}$ & $5.24^{\mathrm{n}}$ & $36.2^{\mathrm{j}}$ & $0.89^{\circ}$ \\
\hline & & MRP & $5.16^{\mathrm{lm}}$ & $0.45^{1}$ & $3.90^{\mathrm{hi}}$ & $44.9^{1}$ & $0.74^{\mathrm{lm}}$ \\
\hline & \multirow{3}{*}{ Monocrop } & CONT & $3.99^{\mathrm{abc}}$ & $0.32^{\mathrm{d}}$ & $2.77^{\mathrm{ab}-}$ & $8.7^{\mathrm{a}}$ & $0.45^{\mathrm{a}-}$ \\
\hline & & FYM & $5.40^{\mathrm{no}}$ & $0.43^{\mathrm{k}}$ & $4.32^{\mathrm{k}}$ & $14.8^{\mathrm{de}}$ & $1.05^{\mathrm{r}}$ \\
\hline & & MRP & $4.56^{\mathrm{fg}}$ & $0.38^{\mathrm{gh}}$ & $3.53^{\mathrm{g}}$ & $33.4^{\mathrm{i}}$ & $0.53^{\mathrm{de}}$ \\
\hline \multirow{11}{*}{ IV } & \multirow{3}{*}{ Crop rotation } & CONT & $4.20^{\text {cde }}$ & $0.35^{\mathrm{e}}$ & $2.97^{\mathrm{c}}$ & $10.2^{\mathrm{abc}}$ & $0.72^{\mathrm{kl}}$ \\
\hline & & FYM & $5.68^{\mathrm{pq}}$ & $0.49^{\mathrm{m}}$ & $4.63^{1}$ & $17.4^{\mathrm{ef}}$ & $1.12^{\mathrm{s}}$ \\
\hline & & MRP & $4.80^{\text {hijk }}$ & $0.40^{\mathrm{ij}}$ & $3.78^{\mathrm{h}}$ & $39.1^{\mathrm{k}}$ & $0.87^{\circ}$ \\
\hline & \multirow{3}{*}{ Intercrop } & CONT & $4.53^{\mathrm{fg}}$ & $0.42^{\mathrm{jk}}$ & $3.36^{\mathrm{ef}}$ & $12.8^{\mathrm{cd}^{-}}$ & $0.61^{\mathrm{gh}}$ \\
\hline & & FYM & $6.13^{\mathrm{r}}$ & $0.56^{\circ}$ & $5.24^{\mathrm{n}}$ & $39.1^{\mathrm{k}}$ & $0.93^{\mathrm{p}}$ \\
\hline & & MRP & $5.18^{\operatorname{lm}}$ & $0.46^{1}$ & $4.28^{\mathrm{k}}$ & $48.5^{\mathrm{m}}$ & $0.77^{\mathrm{m}}$ \\
\hline & \multirow{3}{*}{ Monocrop } & CONT & $4.06^{\mathrm{a} b \mathrm{~cd}}$ & $0.32^{\mathrm{d}}$ & $2.93^{\mathrm{c}}$ & $10.7^{\mathrm{a}^{\mathrm{abc}}-}$ & $0.46^{\mathrm{abc}}$ \\
\hline & & FYM & $5.12^{\mathrm{lm}}$ & $0.45^{1}$ & $4.32^{\mathrm{k}}$ & $17.2^{\text {ef }}$ & $1.06^{\mathrm{r}}$ \\
\hline & & MRP & $4.62^{\mathrm{gh}}$ & $0.39^{\text {hi }}$ & $3.54^{\mathrm{g}}$ & $24.9^{\mathrm{gh}}$ & $0.53^{\mathrm{de}}$ \\
\hline & & Mean & 4.79 & 0.403 & 3.75 & 22.91 & 0.754 \\
\hline & & $\mathrm{LSD}_{0.05}$ & 0.19 & 0.018 & 0.12 & 2.25 & 0.039 \\
\hline
\end{tabular}

Note. Cont-control, MRP-Minjingu rock phosphate, FYM-farm yard manure. Means with the same letters within the column are not significantly different $(\mathrm{P}<0.05)$. 
Table 4. Effects of cropping systems and organic inputs on soil nutrient status in tomato at Kiserian

\begin{tabular}{|c|c|c|c|c|c|c|c|}
\hline Season & Cropping system & Organic inputs & $\mathrm{pH}\left(\mathrm{H}_{2} \mathrm{O}\right)$ & $\mathrm{N}(\%)$ & $\mathrm{C}(\%)$ & $\mathrm{P}(\mathrm{ppm})$ & $\mathrm{K}\left(\mathrm{Cmol} \mathrm{kg}^{-1}\right)$ \\
\hline \multirow[t]{9}{*}{$\mathrm{I}$} & \multirow[t]{3}{*}{ Crop rotation } & CONT & $3.86^{\mathrm{bcd}}$ & $0.31^{\mathrm{d}}$ & $2.78^{\mathrm{c}}$ & $10.9^{\mathrm{bc}}$ & $0.60^{\text {hi }}$ \\
\hline & & FYM & $4.86^{\mathrm{lm}}$ & $0.42^{1}$ & $4.12^{\mathrm{k}}$ & $17.4^{\mathrm{f}}$ & $0.93^{\mathrm{q}}$ \\
\hline & & MRP & $4.40^{\mathrm{ghi}}$ & $0.35^{\text {fgh }}$ & $3.36^{\mathrm{g}}$ & $25.2^{\mathrm{h}}$ & $0.73^{\mathrm{m}}$ \\
\hline & \multirow[t]{3}{*}{ Intercrop } & CONT & $3.99^{\mathrm{de}-}$ & $0.24^{\mathrm{b}^{--}}$ & $2.82^{\mathrm{c}^{-}}$ & $10.2^{\mathrm{abc}-}$ & $0.45^{\mathrm{bc}}$ \\
\hline & & FYM & $5.40^{9}$ & $0.59^{\mathrm{p}}$ & $4.40^{1}$ & $31.3^{\mathrm{i}}$ & $1.05^{\mathrm{s}}$ \\
\hline & & MRP & $4.56^{\mathrm{ijk}}$ & $0.36^{\text {fgh }}$ & $3.59^{\mathrm{h}}$ & $38.9^{\mathrm{k}}$ & $0.53^{\text {ef }}$ \\
\hline & \multirow[t]{3}{*}{ Monocrop } & CONT & $3.74^{\mathrm{ab}-}$ & $0.21^{\mathrm{a}}$ & $2.57^{\mathrm{a}}$ & $9.3^{\mathrm{ab}}$ & $0.40^{\mathrm{a}}$ \\
\hline & & FYM & $4.71^{\mathrm{kl}}$ & $0.49^{\mathrm{n}}$ & $3.79^{\mathrm{j}}$ & $14.9^{\mathrm{e}}$ & $0.93^{\mathrm{q}}$ \\
\hline & & MRP & $4.27^{\mathrm{fg}}$ & $0.31^{\mathrm{d}}$ & $3.10^{\mathrm{de}}$ & $21.5^{\mathrm{g}}$ & $0.47^{\mathrm{cd}}$ \\
\hline \multirow[t]{9}{*}{ II } & \multirow[t]{3}{*}{ Crop rotation } & CONT & $3.93^{\text {bcde }}$ & $0.22^{\mathrm{a}}$ & $2.71^{\mathrm{bc}}$ & $8.7^{\mathrm{ab}}$ & $0.64^{\mathrm{ij}}$ \\
\hline & & FYM & $4.95^{\mathrm{mn}}$ & $0.54^{\circ}$ & $4.35^{1}$ & $14.8^{\mathrm{e}}$ & $0.99^{\mathrm{r}}$ \\
\hline & & MRP & $4.50^{\mathrm{hij}}$ & $0.34^{\text {efg }}$ & $3.24^{\mathrm{fg}}$ & $33.3^{\mathrm{ij}}$ & $0.77^{\mathrm{n}}$ \\
\hline & \multirow[t]{3}{*}{ Intercrop } & CONT & $4.12^{\mathrm{ef}}$ & $0.34^{\mathrm{efg}}$ & $3.11^{\mathrm{de}}$ & $10.9^{\mathrm{bc}}$ & $0.54^{\mathrm{efg}}$ \\
\hline & & FYM & $5.19^{\mathrm{op}}$ & $0.47^{1 \mathrm{~m}}$ & $4.60^{\mathrm{m}}$ & $33.3^{\mathrm{ij}}$ & $0.82^{\circ}$ \\
\hline & & MRP & $4.69^{\mathrm{jkl}}$ & $0.39^{\mathrm{jk}}$ & $3.76^{\mathrm{ij}}$ & $41.27^{1}$ & $0.68^{\mathrm{kl}}$ \\
\hline & \multirow[t]{3}{*}{ Monocrop } & CONT & $3.63^{\mathrm{a}}$ & $0.26^{\mathrm{c}}$ & $2.53^{\mathrm{a}}$ & $9.9^{\mathrm{abc}-}$ & $0.51^{\text {def }}$ \\
\hline & & FYM & $4.57^{\mathrm{ijk}}$ & $0.38^{\mathrm{jk}}$ & $4.06^{\mathrm{k}}$ & $15.8^{\mathrm{ef}}$ & $0.78^{\mathrm{n}}$ \\
\hline & & MRP & $4.13^{\mathrm{ef}}$ & $0.33^{\mathrm{e}}$ & $3.02^{\mathrm{d}}$ & $22.8^{\mathrm{g}}$ & $0.64^{\mathrm{jk}}$ \\
\hline \multirow[t]{9}{*}{ III } & \multirow[t]{3}{*}{ Crop rotation } & CONT & $3.97^{\mathrm{de}}$ & $0.22^{\mathrm{a}}$ & $2.74^{\mathrm{c}}$ & $8.9^{\mathrm{ab}}$ & $0.65^{\mathrm{jk}}$ \\
\hline & & FYM & $5.01^{\mathrm{mno}}$ & $0.54^{\circ}$ & $4.40^{1}$ & $15.1^{\mathrm{ef}}$ & $1.00^{\mathrm{r}}$ \\
\hline & & MRP & $4.55^{\mathrm{ijk}}$ & $0.34^{\mathrm{ef}}$ & $3.28^{\mathrm{fg}}$ & $34.1^{\mathrm{j}}$ & $0.78^{\mathrm{n}}$ \\
\hline & \multirow[t]{3}{*}{ Intercrop } & CONT & $4.24^{\mathrm{fg}-}$ & $0.34^{\mathrm{e} \overline{\mathrm{fg}}}$ & $3.07^{\mathrm{de}}$ & $11.1^{\mathrm{bc}-}$ & $0.54^{\mathrm{fg}^{-}}$ \\
\hline & & FYM & $5.34^{\mathrm{pq}}$ & $0.46^{\mathrm{m}}$ & $4.93^{\mathrm{n}}$ & $34.0^{\mathrm{j}}$ & $0.84^{\circ}$ \\
\hline & & MRP & $4.85^{\operatorname{lm}}$ & $0.43^{1}$ & $3.67^{\mathrm{hi}}$ & $42.2^{1}$ & $0.69^{\mathrm{lm}}$ \\
\hline & \multirow[t]{3}{*}{ Monocrop } & $\mathrm{CONT}$ & $3.75^{\mathrm{abc}}$ & $0.30^{\mathrm{d}^{-}}$ & $2.60^{\mathrm{ab}}$ & $8.2^{\mathrm{a}}$ & $0.43^{\mathrm{ab}^{-2}}$ \\
\hline & & FYM & $5.08^{\text {no }}$ & $0.40^{\mathrm{k}}$ & $4.06^{\mathrm{k}}$ & $13.9^{\mathrm{de}}$ & $0.99^{\mathrm{r}}$ \\
\hline & & MRP & $4.29^{\mathrm{fg}}$ & $0.36^{\mathrm{gh}}$ & $3.32^{\mathrm{g}}$ & $31.4^{\mathrm{i}}$ & $0.50^{\mathrm{de}}$ \\
\hline \multirow[t]{11}{*}{ IV } & \multirow[t]{3}{*}{ Crop rotation } & CONT & $3.94^{\text {cde }}$ & $0.33^{\mathrm{e}}$ & $2.79^{\mathrm{c}}$ & $9.6^{\mathrm{abc}}$ & $0.68^{\mathrm{kl}}$ \\
\hline & & FYM & $5.34^{\mathrm{pq}}$ & $0.46^{\mathrm{m}}$ & $4.35^{1}$ & $16.3^{\text {ef }}$ & $1.04^{\mathrm{s}}$ \\
\hline & & MRP & $4.51^{\mathrm{hijk}}$ & $0.38^{\mathrm{ij}}$ & $3.56^{\mathrm{h}}$ & $36.8^{\mathrm{k}}$ & $0.82^{\circ}$ \\
\hline & \multirow[t]{3}{*}{ Intercrop } & CONT & $4.26^{\overline{f g}}$ & $0.39^{\mathrm{jk}}$ & $3.16^{\mathrm{ef}}$ & $12.0^{\mathrm{cd}}$ & $0.57^{\mathrm{gh}^{-1}}$ \\
\hline & & FYM & $5.76^{\mathrm{r}}$ & $0.53^{\circ}$ & $4.93^{\mathrm{n}}$ & $36.7^{\mathrm{k}}$ & $0.88^{\mathrm{p}}$ \\
\hline & & MRP & $4.87^{\mathrm{Im}}$ & $0.43^{1}$ & $4.02^{\mathrm{k}}$ & $45.6^{\mathrm{m}}$ & $0.73^{\mathrm{m}}$ \\
\hline & \multirow[t]{5}{*}{ Monocrop } & CONT & $3.82^{\mathrm{abcd}}$ & $0.30^{\mathrm{d}}$ & $2.75^{\mathrm{c}}$ & $10.1^{\mathrm{abc}}$ & $0.43^{\mathrm{abc}}$ \\
\hline & & FYM & $4.81^{\mathrm{lm}}$ & $0.43^{1}$ & $4.06^{\mathrm{k}}$ & $16.1^{\mathrm{ef}}$ & $1.00^{\mathrm{r}}$ \\
\hline & & MRP & $4.35^{\mathrm{gh}}$ & $0.36^{\mathrm{hi}}$ & $3.32^{\mathrm{g}}$ & $23.4^{\text {gh }}$ & $0.50^{\mathrm{de}}$ \\
\hline & & Mean & 4.506 & 0.376 & 3.525 & 21.54 & 0.708 \\
\hline & & $\mathrm{LSD}_{0.05}$ & 0.178 & 0.017 & 0.113 & 2.11 & 0.037 \\
\hline
\end{tabular}

Note. Cont-control, MRP-Minjingu rock phosphate, FYM-farm yard manure. Means with the same letters within the column are not significantly different $(\mathrm{P}<0.05)$.

\subsection{Effects of Cropping Systems and Organic Inputs on Soil Nutrient Status in Tomato}

In the tomato plots, treatment effects were as well observed in the two sites, with control treatment, performing significantly $(\mathrm{p}<0.05)$ lower compared to FYM and MRP. Soil pH, N, P K and C increased in the different treatments in the following order control $<$ MRP $<$ FYM in the three cropping systems across the four growing seasons at both sites i.e. MRP and FYM application had significant $(\mathrm{p}<0.05)$ effect on soil nutrient contents. In tomato under rotation with chickpea at Kabete for season four, control had; $\mathrm{K} 0.639 \mathrm{Cmol} \mathrm{kg}^{-1}$ while FYM had $0.994 \mathrm{Cmol} \mathrm{kg}^{-1}$ (Table 3). The results of soil analysis in the tomato under cropping systems at Kabete are shown in Table 3. Those of the trial at Kiserian are shown in Table 4. 
Table 5. Comparison of total harvested $\mathrm{N}$ and $\mathrm{P}$ in maize at Kiserian and Kabete

\begin{tabular}{llllll}
\hline Cropping system & Organic inputs & Kabete N $(\%)$ & Grain P $(\mathrm{ppm})$ & Kiserian N $(\%)$ & Grain P $(\mathrm{ppm})$ \\
\hline Intercrop & CONT & 4.89 & 5980 & 5.16 & 7336 \\
& FYM & 6.43 & 6266 & 6.56 & 7564 \\
& MRP & 5.41 & 7228 & 5.45 & 9080 \\
\hline Crop rotation & CONT & 2.58 & 3146 & 2.36 & 2951 \\
& FYM & 3.36 & 3336 & 3.19 & 3128 \\
\hdashline Monocrop & 2.78 & 3847 & 2.59 & 3609 \\
& MRP & 4.43 & 5658 & 4.43 & 5551 \\
& FYNT & 6.07 & 5933 & 6.04 & 5722 \\
\hline
\end{tabular}

Note. Cont-control, MRP-Minjingu rock phosphate, FYM-farm yard manure.

Table 6. Comparison of total harvested $\mathrm{N}$ and $\mathrm{P}$ in tomatoes at Kiserian and Kabete

\begin{tabular}{llllll}
\hline Cropping system & Organic inputs & Kabete $\mathrm{N}(\%)$ & Fruit P $(\mathrm{ppm})$ & Kabete $\mathrm{N}(\%)$ & Fruit P $(\mathrm{ppm})$ \\
\hline Intercrop & CONT & 9.1 & 2031 & 9.3 & 2021 \\
& FYM & 13.6 & 2399 & 13.4 & 2279 \\
& MRP & 11.2 & 2334 & 11.3 & 2430 \\
\hdashline Crop Rotation & CONT & 5.1 & 1062 & 4 & 1056 \\
& FYM & 7.3 & 1239 & 6.5 & 1189 \\
\hdashline & MRP & 6 & 1232 & 5.1 & 1268 \\
\hdashline Monocrop & CONT & 8.3 & 1946 & 7.9 & 1935 \\
& FYM & 12.1 & 2264 & 12.1 & 2179 \\
& MRP & 10.3 & 2253 & 9.7 & 2316 \\
\hline
\end{tabular}

Note. Cont-control, MRP-Minjingu rock phosphate, FYM-farm yard manure.

In both plants (maize and tomato), total $\mathrm{N}$ and $\mathrm{P}$ were lower in control compared to FYM and MRP. Grain and Fruit, $\mathrm{N}$ and $\mathrm{P}$ increased in the different treatments in the following order control $<\mathrm{MRP}<\mathrm{FYM}$ in the three cropping systems across the four growing seasons at both sites. The results of maize grain and tomato fruit analysis are shown in Tables 5 and 6. Organic-based amendments.

Several studies have shown that long-term application of FYM can maintain soil nutrient levels and stimulate different aspects of soil fertility, because FYM ensures the largely constant presence of active microorganisms and the regular dynamics of biomass carbon (Nardi et al., 2004). In line with this concept, our results showed that combination of FYM and MRP with legume integration resulted in a substantial increase of nitrogen, phosphorus, and carbon. To a lesser extent potassium increased also, indicating the importance of adding manure in cropping systems. Presence of $\mathrm{N}$ and $\mathrm{P}$ in the farm yard manure was expected to affect the rate of OC, N and $\mathrm{P}$ mineralization in this experiment. Presence of $\mathrm{N}$ and $\mathrm{P}$ in FYM and $\mathrm{P}$ in phosphate rock might have led to the increase in OC, total $\mathrm{N}$, and soil $\mathrm{P}$. The $\mathrm{pH}$ condition of the manured and MRP applied plots was adequate for optimum mineralization of $\mathrm{N}$ and $\mathrm{P}$.

\subsection{Soil $N$, $P$ and $K$}

Clear-cut changes were observed on soil nitrogen due to influence of treatments (Tables 2-4). Higher soil N value observed under FYM following by MPR. This was due to the inherent $\mathrm{N}$ content of the waste material incorporated and transformation during composting and after application in the soil. The soil $\mathrm{P}$ was found to be influenced by treatments. The soil $\mathrm{K}$ varied from 0.9 to $1.1 \mathrm{Cmol} \mathrm{kg}^{-1}$ in MPR and FYM as compared to the lowest value obtained under control of $0.4 \mathrm{Cmol} \mathrm{kg}^{-1}$. Visible changes were observed in soil nitrogen due to influence of treatments from 2012 to 2013. Higher soil N values were observed under FYM followed by MPR. This was due to the inherent $\mathrm{N}$ content of the waste material incorporated and transformation during decomposition process. Dynamic changes of soil P from the year 2012 to 2013 was found to be influenced by treatments. The soil P recorded in the year 2012 is relatively lower as compared to the year of 2013. However FYM and MRP under Chickpea rotation and intercrops obtained the higher values compared to control in monocrops. 


\subsection{Effect of FYM and MRP on Soil $p H$}

Brodruzzaman et al. (2010) showed that after 3 crop cycles, soil $\mathrm{pH}$ increased after adding poultry manure but did not change in fields applied with inorganic fertilizers. Kisinyo et al. (2012), reported that large inputs of organic matter inputs (FYM) at $100 \mathrm{t} \mathrm{ha}^{-1} \mathrm{year}^{-1}$ and inputs from food factory sludge compost resulted in an increase in soil $\mathrm{pH}$. The same study also observed that unlike $\mathrm{pH}$, electric conductivity, (EC) of soil remained unaffected.

The increase in soil $\mathrm{pH}$ and reduction of soil exchangeable acidity following application of manure and MPR can be attributed to the release of organic acids (during mineralization of manure), which in turn may have suppressed Al content in the soil through chelation (Onwonga et al., 2008). Moreover, MRP when applied in the soil reacts with water leading to the production of $\mathrm{OH}^{-}$ions and $\mathrm{Ca}^{2+}$ ions which displace $\mathrm{H}^{+}$and $\mathrm{Al}^{3+}$ ions from soil adsorption sites resulting in an increase in soil pH (Kisinyo et al., 2012). These findings correspond to those of Adeniyan et al. (2011) who found increased soil pH with application of manure in Nigeria. It was observed that application of manure either solely or combined with P fertilizer and both P fertilizer and lime had a positive effect on soil exchangeable $\mathrm{K}$, and may be attributed to release of $\mathrm{K}$ from the manure. Similar findings were reported by Chimdi et al. (2012).

\subsection{Effect of FYM and MRP on Plant Available Soil P}

The treatments applied i.e. MPR and FYM might have provided favourable condition for phosphatase enzymes in the mineralization of $\mathrm{P}$ in the soil. The increase in available $\mathrm{P}$ in soil samples applied with MPR substantiated the assertion that the P mineralized was not totally from the integration of chickpea. Kisinyo et al. (2012) emphasized that $\mathrm{P}$ availability is strongly correlated with OC. Presence of OC in the soils fertilized with MRP might also have enhanced P mineralization.

Adeniyan et al. (2011) conducted a pot experiment to compare different organic manures with NPK fertilizer for improvement of chemical properties of acid and depleted soils. The authors showed that application of different types of organic manures enhanced soil organic $\mathrm{C}$, total $\mathrm{N}$, available $\mathrm{P}$, exchangeable $\mathrm{K}$ and CEC better than NPK fertilizer in both soils. This indicates that organic manures are better at enhancing soil chemical properties as compared with inorganic fertilizers.

The MRP treatment in chickpea intercrops had the highest $\mathrm{P}$ (51 ppm) observed at Kabete compared to 13.4 and $41 \mathrm{ppm}$ for the control and manure respectively (Table 1). MRP is a P fertilizer and contains $28 \% \mathrm{P}_{2} \mathrm{O}_{5}$ that improved the soil's mineral components. On the other hand FYM, may have improved the soil's structure and hence its ability to hold nutrients. Thus the MPR and manure treatments created ideal conditions for nutrient uptake by the plant and retention in the soil matrix.

These results indicate that a combination of both organic amendments and legume integrations is better at improving soil $\mathrm{P}$ compared to mono crops without treatments. These results are in agreement with those reported by Kathuku et al. (2011) where there was an increase in yield and nutrients in soil that were supplied with manure applications. As regards the P from organic amendments, Kisinyo et al. (2012) reported that compost applications increased plant available $\mathrm{P}$ in the soil. The biosolids-municipal solid waste co-compost, applied once in four years, was also found to effectively supply $\mathrm{P}$ to soil at $0-15 \mathrm{~cm}$ depth. The soil extractable $\mathrm{P}$ increased on average from 7.2 to $86 \mathrm{mg} \mathrm{kg}^{-1}$ in soil with enhanced application rates from 0 to $200 \mathrm{t} \mathrm{ha}^{-1}$ (Zhang et al., 2009). Furthermore, Kisinyo et al. (2012) suggested that 4-year beef cattle manure and composted manure application based on $\mathrm{N}$ needs of corn could eventually result in soil accumulation of $\mathrm{P}$, since the manure or compost N/P ratio is usually smaller than the corn N/P uptake ratio.

Abera et al. (2005) also found higher soil extractable $\mathrm{P}$ with higher application of manure. The soils tested low $\mathrm{P}$ ( $7.54 \mathrm{mg} \mathrm{kg}^{-1}$ ) before the experiment set. It was observed that the levels of P before planting were higher than after harvest under all treatments except for sole manure. The same trend was also observed by Abera et al. (2005) in Ethiopia and attributed it to the higher phosphorus fixation capacity of acid soils and to the uptake by plants.

To alleviate $\mathrm{P}$ deficiency and improve maize performance in the central rift valley highlands of Kenya, application of Minjingu phosphate rock (MPR) is an alternative to the expensive triple super phosphate fertilizer (Onwonga et al., 2013). MPR is the predominant type of phosphate rock (PR) deposit in Eastern Africa with sufficient quantity and reactivity cum potential for direct application (Okalebo et al., 2007). In the study by Onwonga et al. (2013), solubilisation of MPR was achieved by the acidic nature of the study soils ( $\mathrm{pH}$ in water solution $<5.0$ ) and with the application of manure, conditions necessary for PR solubilisation (Okalebo et al., 2007). 
Certain plant species exhibit mechanisms localized in the rhizosphere that allow for efficient use of $\mathrm{P}$ through dissolution of PR (Okalebo et al., 2007). One such crop is chickpea. The legume forms special root structures called cluster or proteoid roots in response to P deficiency (Kisinyo et al., 2012). The roots strongly acidify the surrounding rhizosphere and secrete large amounts of organic acids, mainly citrate and this enables them access sparingly available nutrients such as phosphate (Weisskopf et al., 2011).

\subsection{Effect of FYM and MRP on Soil Organic Carbon}

Jiang et al. (2006) reported that continuous application of FYM and NPK mineral fertilizers increased soil organic matter by 80 and $10 \%$, respectively, over 20 years in south China. FYM was more effective than inorganic fertilizers at increasing the soil organic carbon stock. The level of organic carbon in soil is believed to be a function of the net input of organic residues by the cropping system Jiang et al. (2006).

Soil and crop-management practices such as crop rotation, legume intercrops, residue management, and phosphate rock therefore have a substantial effect on the level of soil organic carbon over time. Lal (2003) reported application of inorganic fertilizer is important to obtaining high yields, but may have little impact on organic carbon concentration unless used in conjunction with legume integration, no-till and residue management. In support to this Dadhich et al. (2011) reported that application of organic inputs such as FYM, in the required amounts significantly increased organic carbon. The quantity and quality of organic inputs affect rate of decomposition hence the more organic inputs with high $\mathrm{N}$ content the higher the decomposition rate. A considerable number of studies, concerning long-term fertility trials, pointed out that soil organic material applications increased the organic carbon stock and, therefore, increased the cation exchange capacity. This effect was due to the high negative charge of organic matter. This is important for retaining nutrients and making them available to plants (Kaur et al., 2008).

In terms of sustainability, only farmyard manure fertilization maintained the total organic carbon level of $40 \mathrm{t} \mathrm{C}$ $\mathrm{ha}^{-1}$, measured in the top soil layers at the start of a 40 -year experiment, while the average total organic $\mathrm{C}$ depletion was $23 \%$ with liquid manure and mixed fertilization treatments, $43 \%$ with mineral fertilizers alone and $51 \%$ in the control (Nardi et al., 2004). Furthermore, the presence of weakly acidic chemical functional groups on organic molecules makes organic matter an effective buffer, as supported by the findings of Kaur et al. (2008). Effect of FYM and MRP on plant available soil nitrogen

The plant available $\mathrm{N}$ is made up of the inorganic components of $\mathrm{NH}_{4}{ }^{+}$or $\mathrm{NO}_{3}{ }^{-}$. Nitrogen is an essential nutrient for plant growth, chlorophyll and protein formation. Since soil $\mathrm{N}$ is mostly organic in nature, $\mathrm{N}$ concentrations in soil increase with increased organic matter contents (Kisinyo et al., 2012). After 18 years of external inputs and residue removal, the amounts of total soil $\mathrm{N}$, extractable $\mathrm{P}$ and $\mathrm{K}$ in the soil were $0.14 \%, 10.9 \mathrm{mg} \mathrm{kg}^{-1}$ and 0.73 $\mathrm{Cmol} \mathrm{kg}^{-1}$, respectively. Of the three inputs (fertilizer, manure and stover) manuring had the only significant effect on total soil $\mathrm{N}(\mathrm{p}<0.001)$ increasing it by $33 \%$ (Kapkayia et al., 1999).

Another study conducted in Turkey to compare the effects of composted tobacco waste (CTW) with FYM on soil physical and chemical properties and yield of lettuce, showed a significant increase in nitrogen in the FYM plots. Repeated long-term applications of organic amendments not only generally increase the size of the soil organic $\mathrm{N}$ pool, but also cause remarkable changes in soil characteristics, that influence $\mathrm{N}$ dynamics and can lead to a residual effect. Habteselassie et al. (2006a) found an $89 \%$ increase in total soil $\mathrm{N}$ content after 5 years when dairy-waste compost were applied at a rate of $200 \mathrm{~kg} \mathrm{~N} / \mathrm{ha}^{-1}$. The organic matter mineralization process increases the amount of ammonium and nitrate in the soil. However, the $\mathrm{NO}_{3}-\mathrm{N}$ is minimally adsorbed by the soil particles because it is very mobile and is susceptible to losses into ground- and surface waters by infiltrating water. Within this context, it is essential to remember that the synchronization of $\mathrm{N}$ supply with crop $\mathrm{N}$ demand, together with a proper application rate, is the best way to avoid the accumulation of soil mineral N, thus reducing the risk of $\mathrm{NO}_{3}-\mathrm{N}$ leaching (Montemurro \& Maiorana, 2008).

\section{Conclusion}

There were increments in soil plant available nutrients (nitrogen, carbon and phosphorus) with application of organic inputs (farm yard manure and Minjingu rock phosphate). This study demonstrated that application of organic amendments in chickpea integrated cropping systems enhanced soil available potassium, extractable phosphorous total nitrogen and organic carbon content. Manures and rock phosphates application improved soil fertility. 


\section{References}

Abera, T., Feyissa, D., \& Yusuf, H. (2005). Effect of inorganic and organic fertilizer on grain yield of maize-climbing bean intercropping and soil fertility in Western Oromiya, Ethiopia (pp. 1-9). Conference on International Agriculture Research for Development, Stuttgart-Hohenheim.

Adeniyan, O. A., Ojo, A. O., Akinbode, O. A., \& Adediran, J. A. (2011). Comparative study of different organic manures and NPK fertilizer for improvement of soil chemical properties and dry matter yield of maize in two different soils. Journal of Soil Science and Environmental Management, 2(1) 9-13.

Amanullah, M. M., Sekar, S., \& Muthukrishnan, P. (2010). Prospects and potential of poultry manure. Asian Journal of Plant Sciences, 9(4), 172. https://doi.org/10.3923/ajps.2010.172.182

Ayeni, L. S. (2010). Effect of cocoa pod ash, NPK fertilizer and their combinations on soil chemical properties and yield of tomato (Lycopersicon lycopersicum) on two soil types. New York Science Journal, 3, 1-10.

Bationo, A., Kihara, J., Vanlauwe, B., Waswa, B., \& Kimetu, J. (2007). Soil organic carbon dynamics, functions and management in West African agro-ecosystems. Agricultural Systems, 94(1), 13-25. https://doi.org/ 10.1016/j.agsy.2005.08.011

Black, C. A. (1965). Methods of Soil Analysis (Part I and II). American Society of Agronomy, M.W.

Bodruzzaman, M., Meisner, C. A., Sadat, M. A., \& Hossain, M. I. (2010). Long-term effects of applied organic manures and inorganic fertilizers on yield and soil fertility in a wheat-rice cropping pattern (pp. 1-6). 19th World Congress of Soil Science, Soil Solutions for a Changing World.

Bonanomi, G., Antignani, V., Capodilupo, M., \& Scala, F. (2010). Identifying the characteristics of organic soil amendments that suppress soilborne plant diseases. Soil Biology and Biochemistry, 42(2), $136-144$. https://doi.org/10.1016/j.soilbio.2009.10.012

Braun, H. M. H., \& Van de Weg, R. F. (1977). Proposal for rating of land qualities (2nd Approximation). Report No. IC 7. Kenya Soil Survey: Nairobi.

Chen, J. (2006, October). The combined use of chemical and organic fertilizers and/or biofertilizer for crop growth and soil fertility (Vol. 16, p. 20). International Workshop on Sustained Management of the Soil-Rhizosphere System for Efficient Crop Production and Fertilizer Use.

Chimdi, A., Gebrekidan, V., Kibret, K., \& Tadesse, A. (2012). Effects of liming on acidity related chemical properties of soils of different land use systems in W. Oromia, Ethiopia West. Journal of Agricultural Sciences, 8(6), 560-567.

Cooperband, L. (2002). Building soil organic matter with organic amendments. Centre for Integrated Agricultural Systems, University of Wisconsin-Madison, USA.

Dadhich, S. K., Somani, L. L., \& Shilpkar, D. (2011). Effect of integrated use of fertilizer P, farm yard manure and biofertilizers on soil properties and productivity of soybean-wheat crop sequence. Journal of Advances in Developmental Research, 2(1), 42-4.

Das, P., Pal, R., \& Bhattacharyya, P. (2012). Temporal variation of soil nutrients under the influence of different organic amendments. Archives of Agronomy and Soil Science, 58(7), 745-757. https://doi.org/10.1080/ 03650340.2010 .540011

Dean, J. A. (1960). Flame photometry. McGraw-Hill, New York.

FAO-UNESCO. (1990). Soil Map of the World. Revised Legend, World Soil Resources Report No. 60. FAO, Rome.

Giller, K. E., Witter, E., Corbeels, M., \& Tittonell, P. (2009). Conservation agriculture and smallholder farming in Africa: the heretics' view. Field Crops Research, 114(1), 23-34. https://doi.org/10.1016/j.fcr.2009.06.017

Habteselassie, M. Y., Miller, B. E., Thacker, S. G., Stark, J. M., \& Norton, J. M. (2006a). Soil nitrogen and nutrient dynamics after repeated application of treated dairy-waste, Soil Science Society of America Journal, 70, 1328-1337. https://doi.org/10.2136/sssaj2005.0189

Hole, D. G., Perkins, A. J., Wilson, J. D., Alexander, I. H., Grice, P. V., \& Evans, A. D. (2005). Does organic farming benefit biodiversity? Biological Conservation, 122(1), 113-130. https://doi.org/10.1016/j.biocon. 2004.07.018 
Ingram, M. (2007). Biology and Beyond: The Science of Back to Nature Farming in the United States. Annals of the Association of American Geographers, 97(2), 298-312. https://oi.org/10.1111/j.1467-8306.2007. 00537.x

Jama, B., \& Van Straaten, P. (2006). Potential of East African phosphate rock deposits in integrated nutrient management strategies. Anais da Academia Brasileira de Ciências, 78(4), 781-790. https://doi.org/10.1590/ S0001-37652006000400012

Jiang, D., Hengsdijk, H., Dai, T. B., de Boer, W., Jiang, Q., \& Cao, W. X. (2006). Long-term effects of manure and inorganic fertilizers on yield and soil fertility foe a winter-maize system in Jiangsu, China. Pedosphere, 16(1), 25-32.

Kapkiyai, J. J., Karanja, K. N., Qureshi, N. J., Smithson, C. P., \& Woomer, L. P. (1999). Soil organic matter and nutrient dynamics in a Kenyan nitisol under long-term fertilizer and organic input management. Soil Biology and Biochemistry, 31, 1773-1782. https://doi.org/10.1016/S0038-0717(99)00088-7

Kathuku, A. N., Kimani, S. K., Okalebo, J. R., Othieno, C. O., \& Vanlauwe, B. (2011). The effects of integration of organic and inorganic sources of nutrient on maize yield in central Kenya. Innovations as Key to the Green Revolution in Africa, 1, 265-270. https://doi.org/10.1007/978-90-481-2543-2_25

Kaur, T., Brar, B. S., \& Dhillon, N. S. (2008). Soil organic matter dynamics as affected by long-term use of organic and inorganic fertilizers under maize/wheat cropping system, Nutrient Cycling in Agro-ecosystems, 81, 59-69. https://doi.org/10.1007/s10705-007-9152-0

Kisinyo, P. O., Gudu, S. O., Othieno, C. O., Okalebo, J. R., Opala, P. A., Maghanga, J. K., ... Ogola, B. O. (2012). Effects of lime, phosphorus and rhizobia on Sesbania sesban performance in a Western Kenyan acid soil. African Journal of Agricultural Resources, 7(18), 2800-2809. https://doi.org/10.5897/AJAR11.1450

Lal, R. (2003). Carbon sequestration in dry land ecosystems. Environmental Management Journal, 33, 528-544.

Lehmann, J., \& Joseph, S. (Eds.). (2009). Biochar for environmental management: Science and technology. Earthscan.

Makinde, E. A., \& Ayoola, O. T. (2010). Growth, yield and NPK uptake by maize with complementary organic and inorganic fertilizers. African Journal of Food Agriculture and Nutrition Development, 10, 1-15. https://doi.org/10.4314/ajfand.v10i3.54078

Mando, A., Ouattara, B., Somado, A. E., Wopereis, M. C. S., Stroosnijder, L., \& Breman, H. (2005). Long-term effects of fallow, tillage and manure application on soil organic matter and nitrogen fractions and on sorghum yield under Sudano-Sahelian conditions. Soil Use and Management, 21(1), 25-31. https://doi.org/10.1111/j.1475-2743.2005.tb00103.x

Mehlich, A. A., Pinkerton, R. W., \& Kempton, R. (1962). Mass analysis methods for soil fertility evaluation. Internal Publication, Ministry of Agriculture, Nairobi, Kenya.

Miao, Y., Stewart, B. A., \& Zhang, F. (2010). Long-term experiments for sustainable nutrient management in China: A review. Agronomy for Sustainable Development, 31(2), 397-414.

Miles, N., \& Manson, A. (2005). Using manure to supply plant nutrients. Department of Agriculture, Environmental Affairs and Rural Development, Kwazulu Natal, South Africa.

Montemurro, F., \& Maiorana, M. (2008). Organic fertilization as resource for a sustainable agriculture. Fertilizers: Properties, Applications and Effects, 123-146.

Nardi, S., Morari, F., Berti, A., Tosoni, M., \& Giardini, L. (2004). Soil organic matter properties after 40 years of different use of organic and mineral fertilizers. European Journal of Agronomy, 21, 357-367. https://doi.org/ 10.1016/j.eja.2003.10.006

Okalebo, J. R., Gathua, K. W., \& Woomer, P. L. (2002). Laboratory Methods of Soil and Plant Analysis: A working manual. Tropical Soil Biology (2nd ed., p. 128). SSSEA, KARI, Sacred Africa, TSBF-CIAT.

Okalebo, J. R., Woomer, P. L., Othieno, C. O., Karanja, N. K., Ikerra, S., Esilaba, A. O., ... Bationo. A. (2007). The potential of underutilized phosphate rocks for soil fertility replenishment in Africa: Case studies in Western Kenya. African Crop Science Conference Proceedings (Vol. 8, pp. 1589-1598).

Onwonga, R. N., Lelei, J. J., \& Macharia, J. K. (2013). Comparative effects of soil amendments on phosphorus use and agronomic efficiencies of two maize hybrids in acidic soils of Molo County, Kenya. American Journal of Experimental Agriculture, 3(4), 939-958. https://doi.org/10.9734/AJEA/2013/4380 
Onwonga, R. N., Lelei, J. J., Freyer, B., Friedel, J. K., Mwonga, S. M., \& Wandhawa, P. (2008). Low cost technologies for enhancing $\mathrm{N}$ and $\mathrm{P}$ availability and maize (Zea mays L.) performance on acid soils. World Journal of Agricultural Sciences, 4, 862-873.

Payne, R. W., Murray, D. A., Harding, S. A., Baird, D. B., \& Soutar, D. M. (2006). GenStat for Windows (9th ed.). VSN International, Hemel Hempstead.

Peters, J. B., Laboski, C. A. M., \& Bundy, L. G. (2008). Sampling Soils for testing. University of Wisconsin.

Ramesh, P., Singh, M., \& Rao, A. S. (2005). Organic farming: Its relevance to the Indian context. Current Science, 88(4), 561-568.

Riley, H., Pommeresche, R., Eltun, R., Hansen, S., \& Korsaeth, A. (2008). Soil structure, organic matter and earthworm activity in a comparison of cropping systems with contrasting tillage, rotations, fertilizer levels and manure use. Agriculture, Ecosystems \& Environment, 124(3), 275-284. https://doi.org/10.1016/j.agee. 2007.11.002

Rufino, M. C., Rowe, E. C., Delve, R. J., \& Giller, K. E. (2006). Nitrogen cycling efficiencies through resource-poor African crop-livestock systems. Agriculture, Ecosystems \& Environment, 112(4), 261-282. https://doi.org/10.1016/j.agee.2005.08.028

Schnier, H. F., Recke, H., Muchena, F. N., \& Muriuki, A. W. (1996). Towards a practical approach to fertilizer recommendations for food crop production in smallholder farms in Kenya. Nutrient Cycling in Agroecosystems, 47(3), 213-226. https://doi.org/10.1007/BF01986276

Smalling, E. M. A., Nandwa, S. M., \& Janssen, B. H. (1997). Soil fertility in Africa is at stake. In R. J. Buresh, P. A. Sanchez, \& F. Calhoun (Eds.), Replenishing Soil Fertility in Africa (pp. 47-61).

Sombroek, W. G., Braun, H. M. H., \& van der Pouw, B. J. A. (1982). The Exploratory Soil Map and Agro-climate Zone Map Of Kenya (1980) scale 1:1,000,000. Nairobi: Exploratory Soil Survey Report E1, Kenya Soil Survey.

Steel, R. G. D., \& Torrie, J. H. (1987). Principles and Procedures of Statistics. A Biometrical Approach. McGraw Hill, London.

Teng, Y., \& Timmer, V. R. (1994). Nitrogen and phosphorus interactions in an intensively managed nursery soil-plant system. Soil Science Society of America Journal, 58, 232-238. https://doi.org/10.2136/sssaj1994. $03615995005800010035 \mathrm{x}$

Tunstall, B. R. (2008). Global Implications of Soil Organic Matter.

Van Straaten, P. (2002). Rocks for crops: Agrominerals of sub-Saharan Africa (p. 338). Nairobi: ICRAF.

Vanlauwe, B., Diels, J., Sanginga, N., \& Merckx, R. (2005). Long-term integrated soil fertility management in South-western Nigeria: crop performance and impact on the soil fertility status. Plant and Soil, 273(1-2), 337-354. https://doi.org/10.1007/s11104-005-0194-2

Weisskopf, L., Heller, S., \& Eberl, L. (2011). Burkholderia species are major inhabitants of white lupin cluster roots. Applied Environmental Microbiology, 77(21), 7715-7720. https://doi.org/10.1128/AEM.05845-11

Zhang, C. X., Ho, S. C., Chen, Y. M., Fu, J. H., Cheng, S. Z., \& Lin, F. Y. (2009). Greater vegetable and fruit intake is associated with a lower risk of breast cancer among Chinese women. International Journal of Cancer, 125(1), 181-8. https://doi.org/10.1002/ijc.24358

\section{Copyrights}

Copyright for this article is retained by the author(s), with first publication rights granted to the journal.

This is an open-access article distributed under the terms and conditions of the Creative Commons Attribution license (http://creativecommons.org/licenses/by/4.0/). 